\section{SOLUTION OF A TIME-VARYING WIENER FILTERING PROBLEM}

The problem is considered of optimally estimating a signal $s(t)$ when measurements $z(t)=s(t)+n(t)$ are available, $n(t)$ $s(t)$ when measurenents $2(t)=-s(t)+h(t)$ are avaliable, $m(t)$ denoting white noise. The covarances of $s(r)$ and $m(l)$ are known, thus distinguishing the problem formulation from that of Kalman and Bucy, where knowledge is assumed of a
dynamical system generating $s(t)$ when driven by white noise.

It is often assumed that the filtering theory of Kalman and Bucy ${ }^{1}$ is nothing more than the Wiener theory ${ }^{2}$ extended to cover time-varying systems, nonstationary stochastic processes and initial times that are not necessarily minus infinity. Although the general truth of such an assumption will usually be acknowledged, it is important to realise that there are situations where the parallels are not complete. One such situation is considered here.

We assume given measurements $z(t)$ of a signal $s(t)$ corrupted by independent white noise $n(t)$; thus

$$
z(t)=s(t)+n(t)
$$

In eqn. $1, z(\cdot), s(\cdot)$ and $n(\cdot)$ are all $m$ vectors. The noise has zero mean and covariance

$$
E\left\{n(t) n^{\prime}(\tau)\right\}=R(t) \delta(t-\tau)
$$

where it is assumed that $R(t)$ is positive-definite for all $t$. Both the theories referred to above examine the problem of optimally estimating $s(t)$ given measurements $z(\tau), t_{0} \leqslant \tau \leqslant t$, and in both cases information about $s(t)$ is known.

The Wiener theory generally specifies a power spectral density for $s(t)$, whereas the Kalman-Bucy theory generally specifies a linear finite-dimensional dynamic system which, when driven by white noise, has output $s(t)$. Knowledge of this system allows calculation of the covariance of $s(t)$, but knowledge of the covariance of $s(t)$ above does not readily lead to a knowledge of the dynamical system. Yet knowledge of this dynamical system is necessary to apply the KalmanBucy theory.

The conclusion is that the natural generalisation of the Wiener problem, namely the estimation of $s(t)$ given $z(t)$, $E\left\{s(t) s^{\prime}(\tau)\right\}$ and $E\left\{n(t) n^{\prime}(\tau)\right\}$ is only solvable by the standard Kalman-Bucy procedures if one can solve the time-varying spectral-factorisation problem; i.e. if one can pass from a covariance to a system generating that covariance when driven by white noise.

The difficulty of the time-varying spectral-factorisation problem has been known for a long time (see, for example, Reference 3 for a survey of earlier attempts). Among more recent results in the area, Reference 4 can be consulted; this Reference reduces the spectral-factorisation problem under certain circumstances to solving a matrix Riccati equation, evidently indicating that the problem is of similar difficulty to the optimal-filtering problem.

In this letter, we shall show how the spectral-factorisation procedure and the standard Kalman-Bucy filtering theory can be bypassed with the aid of the whitening-filter concept. ${ }^{5}$ Complete solution of the optimal-filtering problem as hitherto discussed requires the solving of two separate problems: spectral factorisation and design of a Kalman-Bucy filter. Use of the whitening filter allows design of an optimal filter with no more difficulty than is required in solving either the spectral-factorisation problem or the Kalman-Bucy filter problem.

We assume that the covariance of $s(\cdot)$ is given as

$$
E\left\{s(t) s^{\prime}(\tau)\right\}=A^{\prime}(t) B(\tau) l(t-\tau)+B^{\prime}(t) A(\tau) l(\tau-t)
$$

where $A(\cdot)$ and $B(\cdot)$ are $n \times m$ matrix functions of time. The separability of the functions multiplying $l(t-\tau)$ and $l(\tau-t)$ corresponds to an assumption that $s(\cdot)$ is generated by driving some unspecified linear, finite-dimensional, dynamical system with white noise.

Because the noise $n(\cdot)$ is independent of $s(\cdot)$,

$$
\begin{aligned}
E\left\{z(t) z^{\prime}(\tau)\right\} & =R(t) \delta(t-\tau)+A^{\prime}(t) B(\tau) l(t-\tau) \\
& +B^{\prime}(t) A(\tau) l(\tau-t) . . \quad . \quad .
\end{aligned}
$$

Let us now distinguish between various filtering problems in terms of the time intervals over which filtering is required; we shall consider three possible time intervals: $\left[t_{0}, T\right],\left[t_{0}, \infty\right)$ and $(-\infty, \infty)$, where $t_{0}$ and $T$ are fixed values of time. For the infinite-interval problem, it proves useful to rewrite eqn. 4 as

$$
\begin{gathered}
E\left\{z(t) z^{\prime}(\tau)\right\}=R(t) \delta(t-\tau)+H^{\prime}(t) \Phi(t, \tau) K(\tau) l(t-\tau) \\
+K^{\prime}(t) \Phi^{\prime}(\tau, t) H(\tau)
\end{gathered}
$$

where $\Phi(\cdot, \cdot)$ is the transition matrix of $\dot{x}=F x$ and $F$ is either completely arbitrary or satisfies certain properties (e.g. boundedness), but is otherwise arbitrary. Procedures for rewriting eqn. 4 in the form of eqn. 5 are well known; we shall, however, have occasion to refer subsequently to the methods of Silverman. ${ }^{6, *}$ Note that eqn. 4 is itself a special case of eqn. 5, corresponding to $\Phi(t, \tau)=I$ and $F \cdots 0$.

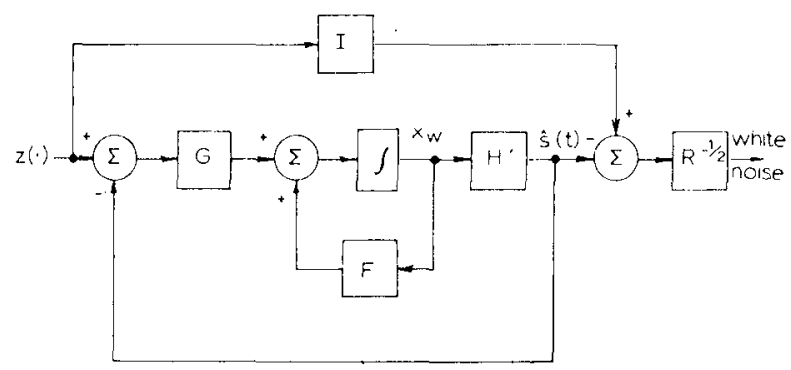

Fig. 1 Whitening filter

The whitening filter for $z(\cdot)$ is a filter whose input is $z(\cdot)$ and whose output is white noise. Such a filter is shown in Fig. 1. The matrices $F$ and $H$ are as in eqn. 5; the matrix $\mathrm{G}$ is given by (Reference 5)

$$
G=(K-1 I H) R^{-1}
$$

with $\Pi$ given by

$$
\begin{aligned}
& \text { II }==\mathrm{II}\left(F^{\prime} \cdots H R^{-1} K^{\prime}\right)-\left(F-K R^{-1} H^{\prime}\right) \mathrm{II} \\
& +\mathrm{II} H R^{-1} H^{\prime} \mathrm{II}+K R^{-1} K^{\prime} \text {. } \\
& \Pi\left(t_{0}\right)=0
\end{aligned}
$$

The initial state $x_{w}\left(t_{0}\right)$ of the whitening filter is zero.

With $F, G$ and $H$ bounded on $\left[t_{0}, T\right]$, existence of a solution to eqn. $7 a$ is guaranteed (Reference 5). If $F, G, H, R$ and $R^{-1}$ are bounded on $\left[t_{0}, \infty\right)$ [or $\left.(-\infty, \infty)\right], F$ is asymptotically stable and $\left[F, H^{\prime}\right]$ is uniformly completely observable, the solution of eqn. $7 a$ exists and is bounded for all time, and the whitening filter is asymptotically stable. ${ }^{5}$

Note that, with the procedures of Silverman, ${ }^{6, *}$ passage from eqn. 4 to eqn. 5 with $F$ bounded, asymptotically stable etc. is possible, provided that $A(\cdot)$ and $B(\cdot)$ satisfy certain conditions presented explicitly elsewhere.*

In Reference 7 the following very important fact is established: The state $x_{w}(t)$ of the whitening filter is the optimal estimate of the state $x(t)$ of the dynamical system

$$
\begin{aligned}
& \dot{x}=F x+G u \\
& y=H^{\prime} x .
\end{aligned}
$$

with $E\left\{u(t) u^{\prime}(\tau)\right\}=I \delta(t \quad \cdots-\tau)$; moreover $E\left\{y(t) y^{\prime}(\tau)\right\}$ $=E\left\{s(t) s^{\prime}(\tau)\right\}$.

As a consequence, the signal labelled $s(t)$ in the Figure is a best estimate of $s(t)$, and the filtering problem is thereby solved.

In summary, the estimation of a noisy signal has been considered where the covariances of the noise and uncontaminated signal have been individually specified. Problem specialisations require the noise to be white and the signal to be derived from the output of a finite-dimensional system excited by white noise. On employing the concept of a whitening filter, it is found that the optimal estimate of the signal is present within the whitening filter, the derivation of which requires the solution of a matrix Riccati equation.

Finally, note that the direct feedthrough block' $I$ and multiplier $R^{-1 / 2}$ of the Figure are unrequired for the determination of $s(t)$.

Application of the ideas presented above is made in 
Reference 8 , where the problem is considered of optimally estimating the differential coefficient of a signal; the measurement of the signal includes corrupting noise.

B. D. O. ANDERSON

20th November 1967

J. B. MOORE

Department of Electrical Engineering

University of Newcastle

Newcastle, NSW2308, Australia

\section{References}

1 KALMAN, R. E., and BUCY, R. C.: 'New results in linear filtering and prediction theory', Trans. ASME, 1961, 83, [D], pp. 95-108

WIENER, N.: 'Extrapolation, interpolation and smoothing of stationary time series' (MIT Press, Cambridge, Massachusetts, 1949)

3 ZaDEH, L. A.: 'Time-varying networks, I', Proc. Inst. Radio Engrs., 1961, 49, pp. 1488-1503

4 LOO, S. G., MOORE, J. B., and ANDERSON, B. D. O.: 'Time-varying spectral EE-6701, Department of Electrical Engineering, University of Newcastle, Australia, June 1967

5 MOORE, J. B., and ANDERSON, B. D. o.: 'Whitening filters: A state-space viewpoint', Technical report EE-6707, Department of Electrical Engineering, University of Newcastle, Australia, August 1967

6 SILVERMAN, L. M.: 'Stable realization of impulse response matrices', IEEE Internat. Convention Record, 1967, pp. 32-36

7 ANDERSON, B. D. O., and MOORE, J. B.: 'State estimation via the whitening filter', Technical report EE-6712, Department of Electrical Engineering, University of Newcastle, Australia, October 1967

8 MOORE, J. B.: 'Optimum differentiation of random signals corrupted by noise', Technical report EE-6715, Department of Electrical Engineering, University of Newcastle, Australia, November 1967 